\title{
Ovarian Cyst Formation Following Gonadotropin-Releasing Hormone-Agonist Administration Decreases the Oocyte Quality in IVF Cycles
}

\author{
Özlem Gün Eryılmaz' , Esma Sarıkaya1, Fatma Nur Aksakal², Sevtap Hamdemir', Muammer Doğan', Leyla Mollamahmutoğlu \\ 'IVF Unit, Zekai Tahir Burak Women Hospital, Ankara, Turkey \\ ${ }^{2}$ Department of Public Health, Faculty of Medicine, Gazi University, Ankara, Turkey
}

\section{ABSTRACT}

Objective: The gonadotropin-releasing hormone-agonist (GnRH-a) treatment during in vitro fertilization (IVF) sometimes causes a functional ovarian cyst during the administration period before gonadotropin stimulation, as an undesired event. The aim of this study was to analyze the effect of these cysts on the IVF outcomes.

Materials and Methods: Out of 981 IVF cycles, 78 with ovarian cysts were retrospectively analyzed with respect to the demographic characteristics, hormonal outcomes, and fertilization, implantation and clinical pregnancy rates.

Results: The metaphase II oocyte ratio, fertilization rate and percentage of high quality embryos (grade 1) were significantly higher in the cyst-negative group ( $p<0.0001 ; p<0.0001 ; p \leq 0.05)$. These same three parameters were also significantly higher in the cyst-aspirated group ( $<<0.01 ; p<0.05 ; p<0.05$ ). Cyst diameters of the aspiration group were significantly higher $(p<0.05)$. No statistically significant differences in implantation and clinical pregnancy rates were determined between the groups.

Conclusion: An ovarian cyst formation during the $\mathrm{GnRH}$-a suppression period negatively affects oocyte quality. Cyst aspiration before gonadotropin stimulation does not improve the IVF outcome.

Key Words: Ovarian cyst, in vitro fertilization (IVF), gonadotropin-releasing hormone agonist

\section{Introduction}

Gonadotropin-releasing hormone-agonist ( $\mathrm{GnRH}-\mathrm{a})$ treatment is one of the main pathways in the long agonist protocol of in vitro fertilization (IVF) treatments. In addition to the many advantages of $\mathrm{GnRH}-a$, it sometimes causes a functional ovarian cyst during the administration period as an undesired event. Such ovarian cysts are still being discussed in terms of their proper management and their impact on the IVF outcome. Mechanical pressure on the ovarian tissue that reduces the area for growing follicles and the decreased blood circulation in and around the follicles are physical explanations for their negative impacts (1-4). Another likely problem concerning these ovarian cysts may be endocrinological in nature. Such cysts may impair folliculogenesis in such a way as to decrease the pre-ovulatory follicle numbers and their quality $(2,3)$. In addition to the studies showing the negative effect of the cysts that appear during $\mathrm{GnRH}-\mathrm{a}$ administration, other studies have concluded that the functional cysts have no effect on the IVF outcome (5-11).

In this study, we aimed to analyze the ovarian cysts in IVF patients, which were found at the end of the GnRH-a ad- ministration period, with respect to their effects on the cycle outcome.

\section{Material and Methods}

Nine hundred and thirty-one patients completed the IVF and intracytoplasmic sperm injection (ICSI) programs between March 2008 and January 2010 in Zekai Tahir Burak Women's Education and Research Hospital, Infertility Research and Treatment Center. Among the 931 patients, 78 with ovarian cysts $\geq 10 \mathrm{~mm}$ in diameter (3) at the end of the GnRH-a suppressive period of the proceeding IVF cycle comprised the study group. Of these 78 patients with ovarian cysts, 46 continued the cycle without cyst aspiration, while the remaining 32 patients with ovarian cysts underwent transvaginal ovarian cyst aspiration (gonadotropin stimulation was administered the day after aspiration in these patients). Patients with aspirated and non-aspirated ovarian cysts were compared with a control group of 163 patients who had no ovarian cysts at any time in their cycle.

The IVF treatment consisted of the long agonist protocol, in which leuprolide acetate (Lucrin, Abbott, Germany) was 
used in the mid-luteal phase of the preceding cycle for pituitary down regulation. Controlled ovarian hyperstimulation was started on the $2^{\text {nd }}$ or $3^{\text {rd }}$ cycle days with human-derived follicle-stimulating hormone (FSH) (Menogon, Ferring Pharmaceuticals, Germany) or recombinant FSH (Puregon, NV Organon, The Netherlands or Gonal-F, Merck Serono, Germany). Gonadotropin administration was continued until the appearance of at least three follicles $\geq 16 \mathrm{~mm}$ on serial ultrasound examinations. Endometrial thickness was measured in the fundal longitudinal axis transvaginally. Ovulation was triggered with $0.25 \mu \mathrm{g}$ recombinant human chorionic gonadotropin (hCG) (Ovitrelle, Merck Serono, Germany). Transvaginal-guided oocyte retrieval was done under general anaesthesia 36 hours after the hCG injection. The morphological grading of the oocytes was done according to the oocyte-cumulus complex, and embryo transfer was done between the $2^{\text {nd }}$ to $5^{\text {th }}$ days.

This study was approved by the Local Ethical Committee of Zekai Tahir Burak Women's Education and Research Hospital.

\section{Statistical analysis}

Statistical analysis was carried out using the Statistical Package for the Social Sciences software (SPSS, version 11.0). All variables were tested for normal distribution with KolmogorovSmirnov test, histogram, and P-P plots. Variables were compared with independent samples t-test or Mann-Whitney $U$ test. All categorical variables were compared with Pearson chisquare and Fisher's exact tests. A p value $<0.05$ was considered as indicating the level of statistical significance.

\section{Results}

The demographic data of the women with basal ovarian cysts in the proceeding IVF cycle and the control group of women without ovarian cysts are presented in Table 1. The causes of infertility were categorized as unexplained, male and tubal factors. Unexplained infertility was the most common factor among the women with ovarian cyst $(52.6 \%)$ and in the control group (58.3\%).

Basal hormonal profiles and outcomes of the patients with ovarian cysts and of the control group are summarized in Table 2. There were no statistically significant differences in Day $3 \mathrm{FSH}$, luteinizing hormone (LH) and E2 levels, the duration of stimulation, and the amount of total gonadotropin used (IU) between the patients with and without ovarian cysts. Of the 78 patients with ovarian cysts, 32 (41\%) underwent cyst aspiration procedure. Cyst diameters in the aspirated and the non-aspirated groups ranged between $1850 \mathrm{~mm}$ (mean \pm SD: $29.0 \pm 8.6$ ) and $10-30 \mathrm{~mm}$ (mean $\pm \mathrm{SD}$ : $18 \pm 5.1$ ), respectively, and the difference was statistically significant $(p=0.0001)$. E2 levels on the day of hCG administration were significantly

Table 1. Demographic characteristics of IVF patients with and without ovarian cysts

\begin{tabular}{|lcc|}
\hline & $\begin{array}{c}\text { Patients with } \\
\text { Ovarian cysts } \\
(\mathbf{n = 7 8 )}\end{array}$ & $\begin{array}{c}\text { Patients without } \\
\text { Ovarian cysts } \\
(\mathbf{n}=163)\end{array}$ \\
\hline Age(years) & $31.2 \pm 4.5$ & $30.6 \pm 4.6$ \\
Infertility duration & $8.1 \pm 4.3$ & $8.6 \pm 4.5$ \\
(years) & & \\
Causes of infertility & $41 / 78(52.6 \%)$ & $95 / 163(58.3 \%)$ \\
Unexplained & $30 / 78(38.5 \%)$ & $62 / 163(38 \%)$ \\
Male factor & $7 / 78(9 \%)$ & $6 / 163(3.7 \%)$ \\
Tubal factor & & \\
\hline Values are expressed as mean $\pm S D$ & \\
\hline
\end{tabular}

Table 2. Patient characteristics and IVF outcomes of the cycles with and without ovarian cysts

\begin{tabular}{|c|c|c|c|}
\hline & $\begin{array}{l}\text { Patients with ovarian cyst } \\
\qquad(n=78)\end{array}$ & $\begin{array}{l}\text { Patients witout ovarian cyst } \\
\qquad(n=163)\end{array}$ & $p$ value \\
\hline Basal E2 levels (pg/mL) & $45.8 \pm 22.4$ & $42.7 \pm 40$ & NS \\
\hline D3 FSH (mIU/mL) & $6.8 \pm 2.0$ & $6.9 \pm 1.9$ & NS \\
\hline D3 LH (mlU/mL) & $5.5 \pm 3.1$ & $5.6 \pm 3.0$ & NS \\
\hline Total gonadotropin used (IU) & $2506.8 \pm 1378.2$ & $2506.8 \pm 1378.2$ & NS \\
\hline Stimulation duration (days) & $10.7 \pm 2.2$ & $10.5 \pm 1.8$ & NS \\
\hline HCG E2 & $2222.1 \pm 1006.1$ & $1996.1 \pm 1113.8^{\star}$ & 0.05 \\
\hline HCG endometrium & $10.3 \pm 1.9$ & $10.2 \pm 1.9$ & NS \\
\hline No. of follicles $\geq 16 \mathrm{~mm}$ & $6.0 \pm 3.0$ & $5.1 \pm 3.1$ & NS \\
\hline No. of oocytes retrieved & $8.0 \pm 5.0$ & $8.9 \pm 6.0$ & NS \\
\hline MIl oocyte ratio & $427 / 630(67.7 \%)$ & $1110 / 1440(77 \%)$ & 0.0001 \\
\hline Fertilization rate & $308 / 630(48.8 \%)$ & $1128 / 1440$ (78.3\%) & 0.0001 \\
\hline No. of embryos transfered & $2.4 \pm 0.7$ & $2.3 \pm 0.8$ & NS \\
\hline Grades I embryo ratio & 139/308 (45.1\%) & $640 / 1128(56.8 \%)$ & 0.05 \\
\hline Implantation rate & $25 / 87(29.5 \%)$ & $64 / 181(35.6 \%)$ & NS \\
\hline Pregnancy rate & $21 / 87(24.3 \%)$ & $53 / 181(29.4 \%)$ & NS \\
\hline
\end{tabular}


higher in women with ovarian cysts $(p<0.05)$. The metaphase II (MII) oocyte number was significantly lower in patients with ovarian cysts $(p<0.05)$. Percentages of MIl oocytes, fertilization rate and the percentage of high quality (Grade 1) embryo transfers were significantly higher in the cyst-negative group $(p<0.05 ; p<0.0001 ; p<0.0001$, respectively). Mll oocyte ratios and the fertilization rate were significantly correlated between the two groups $(p<0.01 ; p<0.05)$.

The cycle characteristics and the outcomes of the patients with and without cyst aspiration are presented in Table 3. The differences in MIl oocyte ratio $(p<0.01)$, fertilization rate $(p<0.05)$, Grade 1 embryo percentage $(p<0.05)$, and cyst diameters $(p<0.05)$ were statistically significant between the two groups.

\section{Discussion}

The appearance of a functional ovarian cyst during the $\mathrm{GnRH}$-a suppression period of the IVF cycle is a controversial issue. The incidence varies between $5.5 \%$ and $53 \%$ in different studies $(1,4,5,8,12)$. In a recent study performed by Qublan et al. (4), the rate was reported as $9.3 \%$ in a large population of IVF patients, which was consistent with our results, at $8.3 \%$.

The mechanism of the cyst formation is not clearly understood. The impressive transient flare-up effect of $\mathrm{GnRH}$-a on immature antral follicles with $\mathrm{LH}$ surge inhibition has been the most frequent explanation (5-8). The presence of an ovarian cyst in IVF cycles has been studied frequently in terms of its effect on the clinical outcomes. Segal et al. (3) pointed out the mechanical effect of the cysts inside the ovary. They suggested that the decreased area in the ovary may impair folliculogenesis and angiogenesis, which was the cause of the poor IVF outcomes. Thatcher et al. (13) stressed the importance of the ovarian cyst found in the follicular phase, which had a more negative effect on the success of ovarian stimulation. Biljan et al. (10) explained the passive diffusion of E2 from the cyst content into the circulation, which increased the basal E2 levels and decreased the basal FSH to false-negative low levels. Our study supported the risk of lower embryo quality in the cyst-positive cycles. IVF patients with cyst occurrence during the $\mathrm{GnRH}$-a administration period had a lower Grade 1 embryo percentage. The higher E2 levels on the hCG administration day in the cyst-positive patients in our study may be explained as in Biljan's study, i.e. passive perfusion of E2 from the cyst mass into the circulation may increase the final E2 level at the end of the stimulation period with an ineffective impact on implantation and the clinical pregnancy rates (10). Keltz et al. (12) reported that ovarian cysts in IVF cycles were seen more in older patients with higher basal FSH levels and lower pregnancy rates. Qublan et al. (4) had also reported the poor IVF outcomes among patients with ovarian cysts. In his study, cycle cancellation rate, the number and quality of the embryos, implantation, and the clinical pregnancy rates were negatively affected. Other studies showed that the pre-stimulation cyst was ineffective $(5,7,11,14)$. Levi et al. (15) showed similar IVF results between the cyst-positive and cyst-negative groups. Kumbak et al. (16) concluded that the pre-stimulation ovarian cyst was not related to poorer pregnancy outcomes and was not an indication to cancel the cycle. Our study also showed similar pregnancy rates between the cyst-positive and cyst-negative groups, but the higher MII oocyte ratio and fertilization rate in the cyst-free patients highlighted the fact that cyst existence in fact has a negative impact on IVF treatments. The better quality of transferred embryos followed the healthier retrieved oocytes in cyst-negative patients. The imbalanced intraovarian environment of cyst-positive ovaries with the physical pressure, disturbed hormonal status or ineffective oxygenation may explain the poorer oocyte results.

Once an ovarian cyst is diagnosed in an IVF patient, the decision ding intervention is problematic. It is still controversial as to whether or not to aspirate the cyst $(3-6,8,10-13)$. The results of many studies were consistent in recommending conservative, non-invasive, non-aspirated treatment of these cysts. Our results showed a significantly higher cyst diameter in the aspirated group. This study was retrospective, and it was ascertained that the larger cysts caused more stress for the physicians, leading to their decision to aspirate. We found that the negative effect of an ovarian cyst on oocytes was more pronounced with a larger cyst, with a worse MII oocyte ratio and lower fertilization rate. The favorable fertilization rate in the non-aspirated group may be due to the existence of a smaller cyst with less ovarian damage or the absence of any direct disturbing impact of the aspiration procedure on the ovaries. Cyst aspiration with intraovarian instrumentation may cause bleeding, impair the perifollicular circulation, or increase the inflammatory chemicals. These probable explanations suggest that aspiration of such cysts should be avoided before stimulation. As the pregnancy rates of the aspirated

Table 3. Cycle characteristics and IVF outcomes of patients with cyst aspiration and non-aspiration

\begin{tabular}{|lccc|}
\hline & Cyst aspirated group ( $\mathbf{n = 3 2})$ & Cyst non- aspirated group ( $\mathbf{n = 4 6 )}$ & $\mathrm{p}$ value \\
\hline Mll oocyte ratio & $179 / 284(63.0 \%)$ & $248 / 346(71.6 \%)$ & 0.01 \\
Fertilization rate & $122 / 284(42.9 \%)$ & $186 / 346(53.7 \%)$ & 0.05 \\
No. of embryos transferred $(\mathrm{n})$ & $2.3 \pm 0.8$ & $2.6 \pm 0.6$ & $\mathrm{NS}$ \\
Grades I embryo ratio & $49 / 122(40.8 \%)$ & $95 / 186(51.5 \%)$ & 0.05 \\
Cyst diameter(mm) & $29.9 \pm 8.6$ & $18.2 \pm 5.1$ & 0.05 \\
Implantation rate & $10 / 38(28.1 \%)$ & $14 / 49(30.4 \%)$ & $\mathrm{NS}$ \\
Pregnancy rate & $6 / 38(17.3 \%)$ & $7 / 49(15.6 \%)$ & $\mathrm{NS}$ \\
\hline Values are expressed as mean \pm SD. NS: Not significant & & \\
\hline
\end{tabular}


and non-aspirated groups were not statistically different, it is once again shown in this analysis that aspiration of an ovarian cyst before gonadotropin administration has no positive impact on the IVF outcome. Further, the aspiration procedure itself may cause psychological stress for the patient, and the risks of anaesthesia may also be problematic; thus, intervention with aspiration does not appear to be mandatory.

In summary, the results of our study support the findings of previous studies in which cyst appearance during the $\mathrm{GnRH}-\mathrm{a}$ administration period was harmful for the ovary. The impressive negative effects were on fertilization, but not on the overall implantation and pregnancy rates. Once a cyst is observed, we do recommend the continuation of the IVF treatment without cyst aspiration in order to prevent the likely ovarian damage.

\section{Conflict of Interest}

No conflict of interest was declared by the authors.

\section{References}

1. Fiszbajn GE, Lipowicz RB, Elberger L, Grabia A, Papier SD, Olmedo SBP, et al. Conservative management versus aspiration of functional ovarian cysts before ovarian stimulation for assisted reproduction. J Assist Reprod Genet 2000;17:260-3. [CrossRef]

2. Feldberg D, Goldman J, Ashkenazi J, Shelef M, Dicker D, Hart J. Ovarian cyst aspiration during induction of ovulation for IVF. Hum Reprod 1987;2:115.

3. Segal $S$, Schifren JL, Isaacson $K B$, Leykin $L$, Chang $Y, P a l ~ L$, et al. Effects of a baseline ovarian cyst on the outcomes of in vitro fertilization-embryo transfer. Fertil Steril. 1999;71:274-7. [CrossRef]

4. Qublan HS, Amarin Z, Tahat YA, Smadi AZ, Kilani M. Ovarian cyst formation following $\mathrm{GnRH}$ agonist administration in IVF cycles: incidence and impact. Hum Reprod 2006;21:640-4. [CrossRef]

5. Feldberg D, Ashkenazi J, Dicker D, Yeshaya A, Goldman GA, Goldman JA. Ovarian cyst formation: a complication of gonadotropinreleasing hormone agonist therapy. Fertil Steril 1989;51:42-5.
6. Herman A, Ron-El R, Golan A, Nahum H, Soffer Y, Caspi E. Follicle cysts after menstrual versus midluteal administration of gonadotropin-releasing hormone analog in in vitro fertilization. Fertil Steril 1990;53:854-8.

7. Jenkins JM, Davies DW, Anthony F, Wood P, Gadd SG, Watson RH, et al. The detrimental influence of functional ovarian cysts during in-vitro fertilization cycles. Hum Reprod 1992;7:776-80.

8. Ron-El R, Herman A, Golan A, Raziel A, Soffer Y, Caspi E. Follicle cyst formation following long acting gonadotropin-releasing hormone analog administration. Fertil Steril 1989;52:1063-6.

9. Tarlatzis BC, Bili H, Bontis J, Lagos S, Vatev I, Mantalenakis S. Follicle cyst formation after administration of different gonadotropinreleasing hormone analogues for assisted reproduction. Hum Reprod 1994;9:1983-6.

10. Biljan MM, Lapensee L, Mahutte NG, Bissonnette F, Hemmings R, Tan SL. Effects of functional ovarian cysts detected on the 7th day of gonadotropin-releasing hormone analog administration on the outcome of IVF treatment. Fertil Steril 2000;74:941-5. [CrossRef]

11. Hornstein MD, Barbieri RL, Ravnikar VA, McShane PM. The effects of baseline ovarian cysts on the clinical response to controlled ovarian hyperstimulation in an in vitro fertilization program. Fertil Steril 1989;52:437-40.

12. Keltz MD, Jones EE, Duleba AJ. Baseline cyst formation after luteal phase gonadotropin releasing hormone agonist administration is linked to poor in vitro fertilization outcome. Fertil Steril 1995;64:568-72.

13. Thatcher SS, Jones E, DeCharney AH. Ovarian cysts decrease the success of controlled ovarian stimulation and in vitro fertilization. Fertil Steril 1989;52:812-6.

14. Firouzabadi RD, Sekhavat $L$, Javedani M. The effect of ovarian cyst aspiration on IVF treatment with GnRH. Arch Gynecol Obstet 2010;281:545-9. [CrossRef]

15. Levi R, Özçakir HT, Adakan S, Göker TEN, Tavmergen E. Effect of ovarian cysts detected on the beginning day of ovulation induction to the success rates in ART cycles. J Obstet Gynecol Res 2003;29:257-61. [CrossRef]

16. Kumbak B, Kahraman S. Management of prestimulation ovarian cysts during assisted reproductive treatments: impact of aspiration on the outcome. Arch Gynecol Obstet 2009;279:875-80. [CrossRef] 